\title{
Dinámica del crecimiento de un bosque templado bajo manejo en el noroeste de México
}

\section{Growth dynamic of a tempered forest under management in the Northwest of Mexico}

\author{
Javier Hernández-Salas',2, Óscar A. Aguirre-Calderón', Eduardo Alanís-Rodríguez, Javier Jiménez-Pérezl, \\ Eduardo J. Treviño-Garza!, Marco A. González-Taglel, Concepción Luján-Álvarez², Jesús M. Olivas-García² y \\ L. Alfonso Domínguez-Pereda ${ }^{3}$
}

Universidad Autónoma de Nuevo León. Facultad de Ciencias Forestales. Linares, Nuevo León, México. 2 Universidad Autónoma de Chihuahua. Facultad de
Ciencias Agrícolas y Forestales. Ciudad Delicias,
Chihuahua, México.
3 Dirección Técnica Forestal, Ejido El Largo y Anexos.
Ciudad Madera, Chihuahua, México.
* Autor de correspondencia.
eduardo.alanisrd@uanl.edu.mx

\section{RESUMEN}

Se analizó la dinámica del elemento arbóreo de bosques naturales de segundo crecimiento de Pinus en el noreste de México. Para ello, a partir de datos previos a las intervenciones silvícolas, se evaluó el comportamiento de diferentes parámetros dasométricos relacionados con la densidad y las dimensiones del arbolado. Se utilizaron registros de todos los individuos con diámetro a la altura del pecho mayor o igual a $7.6 \mathrm{~cm}$, provenientes de tres monitoreos periódicos (años 1986, 1996 y 2006) de parcelas permanentes. A las variables dasométricas se les realizaron pruebas de comparación de medias entre evaluaciones. Las diferencias en dimensiones de los parámetros, en evaluaciones sucesivas, resaltan la dinámica de crecimiento y desarrollo propia de masas regulares. El crecimiento de árboles promedio y parcelas mostró un incremento individual mayor en el primer periodo, que se mantuvo a nivel bosque en el último periodo, lo que sugiere un rendimiento continuo, producto de un sistema de manejo forestal sustentable. En conclusión, el bosque es regular, registra un incremento en las dimensiones del arbolado y el volumen de la masa, y permite sostener la producción maderable con las intervenciones silvícolas aplicadas para su desarrollo.

PALABRAS CLAVE: ejido El Largo, estructura forestal, inventario forestal continuo, parámetros dasométricos, Pinus arizonica Engelm.

\section{ABSTRACT}

The dynamics of the tree component of second-growth natural forests of Pinus in northwestern Mexico was analyzed. For this, the behavior of different dasometric parameters related to the density and dimensions of trees was evaluated, from data obtained previous to silvicultural interventions. Records of all individuals with diameter at breast height $\geq 7.6 \mathrm{~cm}$ were used, from three periodic inventories of permanent plots (1986, 1996 and 2006). Mean test comparisons were carried out for the dasometric parameters among assessments. Differences in dimensions of the parameters in successive evaluations denote the dynamics of growth and development belonging to a regular forest. The average trees and plots growth showed higher individual increment in the first period. This behavior was constant at the forest level in the last period, suggesting a continuous yield, which is produced by a sustainable forest management system. In conclusion, the studied forest is an even-aged forest which records an increment in the size of trees and the volume of the stands, and allows sustained timber production with silvicultural interventions applied for its development.

KEYWORDS: ejido El Largo, forest structure, continuous forest inventory, dasometric parameters, Pinus arizonica Engelm. 


\section{INTRODUCCIÓN}

La dinámica de un bosque se modifica temporalmente cuando el gestor la interviene para hacer un aprovechamiento maderable o para obtener una estructura determinada, debido a la apertura del dosel y extracción de arbolado (Manzanero y Pinelo, 2004). Asimismo, dado que las intervenciones silvícolas determinan la estructura del bosque residual, también condicionan las intervenciones subsecuentes (Aguirre, Hui, Gadow y Jiménez, 2003; Reque, Sarasola, Gyenge y Fernández, 2007), por lo que la conservación de los bosques depende de la calidad del manejo forestal (McGinley y Finegan, 2001; Bell et al., 2014). Por lo tanto, conocer la dinámica de crecimiento y la estructura horizontal y vertical de un bosque contribuye a validar o replantear su manejo silvícola y aprovechamiento (Manzanero y Pinelo, 2004; Reque, et al., 2007; Garcia-Gonzalo, Zubizarreta, Kellomäki y Peltola, 2017). Por otra parte, la caracterización de las poblaciones arbóreas a través del tiempo permite su comparación y conocer el grado de discrepancia en determinado atributo o variable (Aguirre et al., 2003; Fry, 2011; Jiménez, Alanís, González, Aguirre y Treviño, 2013).

Los bosques del ejido El Largo y Anexos (noroeste de México) se han aprovechado en forma ordenada por más de 80 años. En las últimas tres décadas, las áreas de corta se intervienen cada 10 años, con base en los programas de manejo y a la normatividad vigente (SmartWood, 2004). Se presume que este periodo y la intensidad de intervención silvícola son adecuados para mantener y en lo posible incrementar la capacidad productiva maderable, bajo los principios del manejo forestal sustentable.

Sin embargo, en estos bosques como los del resto de México, son escasos los análisis de los efectos del uso antropogénico en la estructura vegetal (Corral, Aguirre, Jiménez y Corral, 2005; Návar y González, 2009; Hernández-Salas et al., 2013; López et al., 2017; Graciano-Ávila, Aguirre-Calderón, Alanís-Rodríguez y Lujan-Soto, 2017). Por lo anterior, se consideró necesario evaluar el crecimiento mediante la valoración de algunos parámetros dasométricos y, a partir de la comparación de sus valores antes de la intervención silvícola, conocer la dinámica y estructura del bosque para replantear una gestión forestal productiva y sustentable en el área estudiada.

\section{OBJETIVOS}

Evaluar la dinámica y estructura del crecimiento de un bosque templado bajo manejo, de segundo crecimiento, en el del ejido El Largo y Anexos, Madera, Chihuahua (noroeste de México).

\section{MATERIALES Y MÉTODOS}

\section{Área de estudio}

Se ubica en un bosque templado frío, natural, de segundo crecimiento, denominado área de corta "Moctezuma", en la zona norte del ejido El Largo y Anexos, Madera, Chihuahua, México; en las coordenadas $29^{\circ} 49^{\prime} 41^{\prime \prime}$ a $29^{\circ} 55^{\prime} 34^{\prime \prime}$ latitud norte, y $108^{\circ}$ 13' 28" a $108^{\circ} 18$ '34" longitud oeste. Según datos del Instituto Nacional de Estadística y Geografía [Inegi] (1984), es un bosque maderable de Pinus arizonica, con presencia de $P$. durangensis, $P$. engelmannii, P. leiophylla, P. ayacabuite, Quercus sideroxyla, Q. fulva y Juniperus sp., con suelo rocoso y pendiente ligera. La altitud promedio es de $2450 \mathrm{~m}$, el clima semifrío, subhúmedo, con verano fresco largo y la precipitación media anual es de $700 \mathrm{~mm}$ (Comisión Nacional para el Conocimiento y Uso de la Biodiversidad [Conabio], 1998).

\section{Método de muestreo}

En 1986, dentro del proyecto de Inventario Forestal Continuo del ejido, previo a la corta, se establecieron 46 parcelas circulares permanentes de 0.1 ha, con una distribución sistemática y una intensidad de muestreo de $0.2 \%$. En estas, se inventariaron y enumeraron todos los árboles con diámetro (a $1.3 \mathrm{~m}$ de altura) mayor o igual a $7.6 \mathrm{~cm}$, para ellos se registró especie, diámetro y altura total. Las parcelas se midieron en intervalos de 10 años (1996 y 2006), antes de cada intervención silvícola.

\section{Tratamiento de los datos}

Densidad. Este parámetro y cinco más se determinaron y analizaron para cada parcela. El número de árboles $(N)$ por unidad de área (ha) es probablemente la evaluación estructural más básica y utilizada como medida de densidad (Bettinger, Boston, Siry y Grebner, 2009).

Área basal. Es una variable muy importante para la caracterización de un bosque (Prodan, Peters, Cox y Real, 1997); es una medida del arbolado ampliamente usada en la dasonomía 
y es la relación entre las secciones normales de los árboles de un espacio forestal y la superficie de terreno que ocupan (Bettinger et al., 2009). A partir de los datos de diámetro, se calculó el área basal individual [1a] y de la parcela, proyectada a una hectárea [1b].

$g=(d)^{2} \times 0.7854$

$$
\mathrm{G}=\left(\sum \mathrm{g}_{\mathrm{i}}\right) \cdot 10
$$

Donde:

$\mathrm{g}=$ área basal $\left(\mathrm{m}^{2}\right)$ de un árbol dado (i)

$\mathrm{d}=$ diámetro $(\mathrm{m})$ a $1.3 \mathrm{~m}$ de altura

$\mathrm{G}=$ área basal de la parcela $\left(\mathrm{m}^{2}\right)$, multiplicada por 10, área basal por hectárea $\left(\mathrm{m}^{2} \cdot \mathrm{ha}^{-1}\right)$.

Diámetro cuadrático. Representa el diámetro del árbol de área basal promedio del rodal (Bettinger et al., 2009). Es fundamental para rodales de distribución diamétrica regular y es el más utilizado para caracterizar un rodal (Prodan et al., 1997). Se calculó con el área basal y número de árboles [2].

$$
\operatorname{Dg}=\sqrt{\frac{4}{\pi} \cdot \frac{\mathrm{G}}{\mathrm{N}}}
$$

Donde:

$\mathrm{Dg}=$ diámetro medio cuadrático $(\mathrm{m})$

$\mathrm{G}=$ área basal $\left(\mathrm{m}^{2}\right)$

$\mathrm{N}=$ número de árboles de la parcela

$\pi=$ constante igual a 3.1416

Altura media. Caracteriza al conjunto de la masa del arbolado y permite comparar el tamaño de los árboles entre rodales y está fuertemente relacionada con la calidad del sitio (Bettinger et al., 2009). Es utilizada como altura de entrada a las tablas de rendimiento (Prodan et al., 1997). Se estimó con la fórmula de Lorey [3]:

$$
H_{L}=\frac{g_{1} h_{1}+g_{2} h_{2}+\ldots+g_{n} h_{n}}{G}
$$

Donde:

$\mathrm{g}=$ área basal de cierto árbol $\left(\mathrm{m}^{2}\right)$

$\mathrm{h}=$ altura de ese mismo árbol (m)

$\mathrm{n}=$ número de árboles presentes en la parcela

$\mathrm{G}=$ área basal total de la parcela $\left(\mathrm{m}^{2}\right)$
Altura dominante. Para el género Pinus, se consideró la altura de los cinco árboles con mayor diámetro en la parcela, que equivalen a los 50 árboles más gruesos de la hectárea, denominada altura de Assmann modificada (Diéguez-Aranda et al., 2009; Prodan et al., 1997). Para Quercus y Juniperus se tomó la altura del árbol más grueso, denominada altura de Hamilton (Bengoa, 1999). La altura del total se calculó considerando los cinco árboles de mayor diámetro en la parcela, independientemente de la especie y/o género; esta altura, se usa para determinar el índice de sitio (Gadow, Sánchez y Álvarez, 2007) y es la variable básica para los modelos estáticos usados en la construcción de tablas de producción (Diéguez-Aranda et al., 2009).

Volumen. Es la medida más común en un inventario forestal; en un plan de manejo forestal está relacionado con la factibilidad económica de las actividades de gestión forestal (Bettinger, et al., 2009). Se calculó con las ecuaciones de volumen individual que determinó y usa el servicio técnico forestal del ejido El Largo y Anexos. Para el género Pinus se usó la ecuación [4]:

$$
\mathrm{VTA}=0.000074986\left(\mathrm{~d}^{2} \mathrm{H}\right)^{0.956224}
$$

Donde:

VTA $=$ volumen total árbol con corteza $\left(\mathrm{m}^{3}\right)$

$\mathrm{d}=$ diámetro con corteza a la altura de $1.3 \mathrm{~m}(\mathrm{~cm})$

$\mathrm{H}=$ altura total del árbol (m)

En los géneros Quercus y Juniperus se utilizó la ecuación [5]:

$$
\text { VTA }=0.0002186\left(d^{2} H\right)^{0.8737002}
$$

Espaciamiento y espesura. En el manejo de la densidad de un régimen silvícola, los índices de espaciamiento se utilizan para determinar la necesidad e intensidad de un aclareo (Prodan et al., 1997). Una opción es el índice de Hart-Becking [6], que es el cociente o proporción entre el espaciamiento promedio de los árboles de un rodal y su altura dominante (Diéguez-Aranda et al., 2009; Gadow et al., 2007). Este espaciamiento relativo $\left(S_{\%}\right)$ es eficaz para comparar espesuras de masas regulares y su valor es inversamente proporcional a la espesura (Serrada, 2008).

$$
\text { Espesura: } \mathrm{S}_{\%}=(\mathrm{a} / \mathrm{Ho}) \cdot 100
$$


Donde:

$\mathrm{a}=$ espaciamiento medio de los árboles ( $\mathrm{m}$ ), en masas naturales asume una distribución en tresbolillo [7]

Ho = altura dominante de la parcela (m, altura de Assmann)

$$
\text { Espaciamiento: } a=\sqrt{(20000 / N \cdot \sqrt{3})}
$$

Donde:

$\mathrm{N}=$ densidad, el número de árboles por hectárea

En el presente estudio sólo se evaluó para el arbolado total y en Pinus, ya que Quercus y Juniperus no estaban presentes en varias parcelas.

Análisis estadístico. A cada parámetro dasométrico se le constató la distribución normal (prueba de KolmogórovSmirnov) y homocedasticidad de los datos (prueba de Bartlett), para usar el ANOVA de un factor o, en su ausencia, su equivalente: la prueba no paramétrica de Kruskal-Wallis, bajo la hipótesis nula de igualdad de medias o medianas entre evaluaciones, respectivamente. Para las diferencias significativas, se usaron las pruebas correspondientes de comparaciones múltiples, de medias de Tukey y de rangos de Nemenyi (Wheater y Cook, 2005). El nivel de significancia para todas las pruebas fue $\alpha=0.05$. Para realizar los análisis se utilizaron los programas SPSS, PASW statistics 18 y Minitab 15.

\section{Resultados}

\section{Densidad}

La densidad total del arbolado disminuyó significativamente $\left(\mathrm{Chi}^{2}\right.$ = 7.63; g.l. $=2 ; \mathrm{P}=0.022)$ : en el año 1986 , el $\mathrm{N}$ promedio $( \pm$ desviación estándar) fue de 940.7 árboles ${ }^{h} \mathrm{ha}^{-1} \pm 504.5$ árboles $\cdot \mathrm{ha}^{-}$ ${ }^{1}$ y en 2006 descendió a 716.3 árboles $\cdot \mathrm{ha}^{-1} \pm 413.7$ árboles $\cdot \mathrm{ha}^{-1}$, un 23.8\% (Fig. 1A). El género Pinus decreció 18.6\% entre periodos; de esta merma, 86.6\% se dio entre 1996 y 2006, pero la prueba K-W no registró diferencias significativas entre mediciones $\left(\mathrm{Chi}^{2}=2.06\right.$; g.l. $\left.=2 ; \mathrm{P}=0.356\right)$. En 1986 la media fue 845.9 árboles $h^{-1} \pm 539.0$ árboles ${ }^{-1} h^{-1}$ y en 2006, 688.7 árboles $h^{-1} \pm 417.9$ árboles ${ }^{\cdot h a^{-1}}$. El género Quercus mostró diferencias significativas $\left(\mathrm{Chi}^{2}=6.92 ;\right.$ g.l. $\left.=2 ; \mathrm{P}=0.031\right)$, la media de $1986,81.3 \pm 102.1$ árboles ${ }^{\cdot h} a^{-1}$, fue diferente a la de 2006, 20.8 árboles $\cdot \mathrm{ha}^{-1} \pm 22.49$ árboles ${ }^{\cdot h a^{-1}}$, decreció 74.3\%. En Juniperus no se observaron diferencias significativas $\left(\mathrm{Chi}^{2}=0.30\right.$; g.l. $=2 ; \mathrm{P}=$ $0.861)$.

Destaca el incremento proporcional de Pinus y el descenso de Quercus, por efecto de las intervenciones silvícolas. En 1986 constituían $89.9 \%$ y $8.6 \%$ respectivamente y para 2006 representaron $96.15 \%$ y $2.91 \%$ del $\mathrm{N}$ total. Juniperus, con poca variación, participó con $0.94 \%$.

\section{Área basal (G, m2·ha-1)}

El G total incrementó $21.0 \%$ en el primer intervalo; luego decreció $6.4 \%$ en el segundo. Presentó diferencias significativas $(\mathrm{F}=4.255 ;$ g.l. $=2 ; \mathrm{P}=0.016)$ entre 1986 y 1996: ascendió de $19.58 \mathrm{~m}^{2} \cdot \mathrm{ha}^{-1} \pm 6.81 \mathrm{~m}^{2} \cdot \mathrm{ha}^{-1}$ a $23.70 \mathrm{~m}^{2} \cdot \mathrm{ha}^{-1} \pm 7.30 \mathrm{~m}^{2} \cdot \mathrm{ha}^{-1}$; posteriormente descendió a $22.17 \mathrm{~m}^{2} \cdot \mathrm{ha}^{-1} \pm 6.40 \mathrm{~m}^{2} \cdot \mathrm{ha}^{-1}$ en 2006 (Fig. 1B).

El G de Pinus tuvo un incremento de $29.2 \%$ en la primera década, superior al bosque; en la segunda decreció 2.2\%. Esta variable mostró diferencias significativas $(\mathrm{F}=5.719$; g.l. $=2 ; \mathrm{P}=$ 0.004); la media del año $1986\left(16.37 \mathrm{~m}^{2} \cdot \mathrm{ha}^{-1} \pm 7.42 \mathrm{~m}^{2} \cdot \mathrm{ha}^{-1}\right)$ fue estadísticamente menor a las de $1996\left(21.16 \mathrm{~m}^{2} \cdot \mathrm{ha}^{-1} \pm 8.19 \mathrm{~m}^{2} \cdot \mathrm{ha}\right.$ 1) y $2006\left(20.69 \mathrm{~m}^{2} \cdot \mathrm{ha}^{-1} \pm 6.8 \mathrm{~m}^{2} \cdot \mathrm{ha}^{-1}\right)$. Para el género Quercus la prueba K-W no registró diferencias significativas $\left(\mathrm{Chi}^{2}=5.94\right.$; g.l. $=2 ; \mathrm{P}=0.051$ ), aunque el valor de $\mathrm{G}$ decreció 59.4\%, de 1986 a 2006.

\section{Diámetro cuadrático $(\mathrm{Dg}, \mathrm{cm})$}

El Dg total arbóreo incrementó 23.7\% (4.03 cm) entre 1986 y 2006, del cual 55.3\% (2.22 cm) correspondieron al primer intervalo. Mostró incrementos significativos en las tres mediciones $(\mathrm{F}=18.610$; g.l. $=2 ; \mathrm{P}<0.001)$. Los valores promedio fueron $16.99 \mathrm{~cm} \pm 3.18 \mathrm{~cm}, 19.22 \mathrm{~cm} \pm 2.97 \mathrm{~cm} \mathrm{y}$ $21.02 \mathrm{~cm} \pm 3.35 \mathrm{~cm}$, respectivamente (Fig. 1C).

El Dg de Pinus incrementó 25.1\% (4.19cm) entre 1986 y 2006 , del cual $59.9 \%(2.51 \mathrm{~cm})$ correspondió al primer intervalo. Los Dg de Pinus fueron inferiores a los totales en las tres mediciones debido a que los de Quercus fueron superiores. El ANOVA mostró diferencias significativas entre evaluaciones ( $\mathrm{F}$ = 17.540; g.l. = 2; $\mathrm{P}<0.001)$, el Dg promedio de 1986, $16.65 \mathrm{~cm}$ $\pm 3.35 \mathrm{~cm}$, resultó estadísticamente menor a $19.16 \mathrm{~cm} \pm 3.35 \mathrm{~cm}$ y a $20.84 \mathrm{~cm} \pm 3.63 \mathrm{~cm}$, correspondientes a 1996 y 2006 . 
B

A
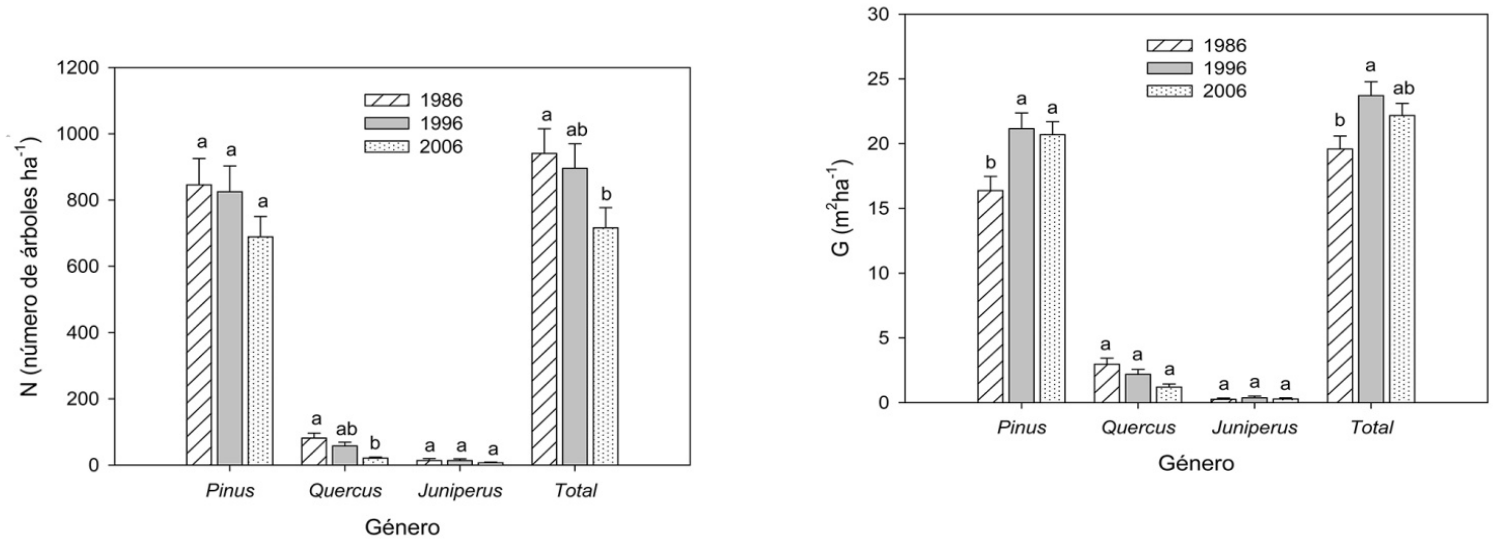

C

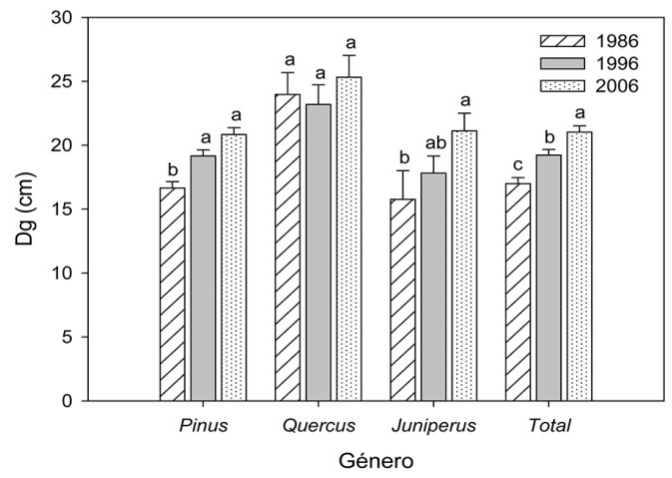

E

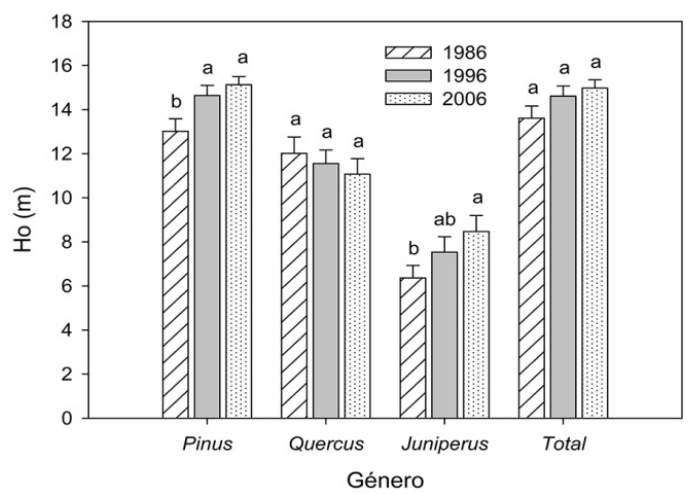

D

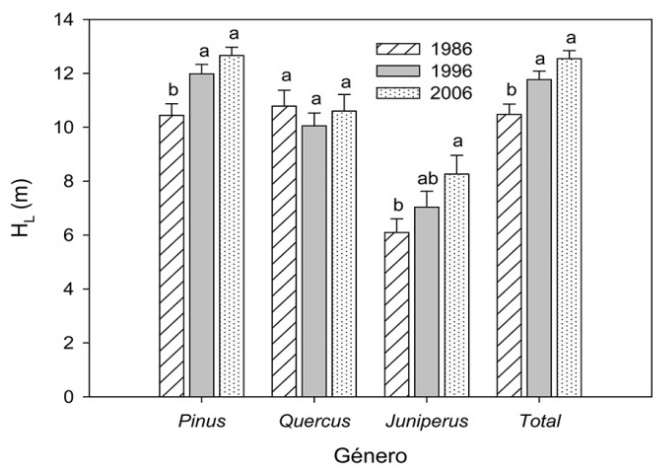

$\mathbf{F}$

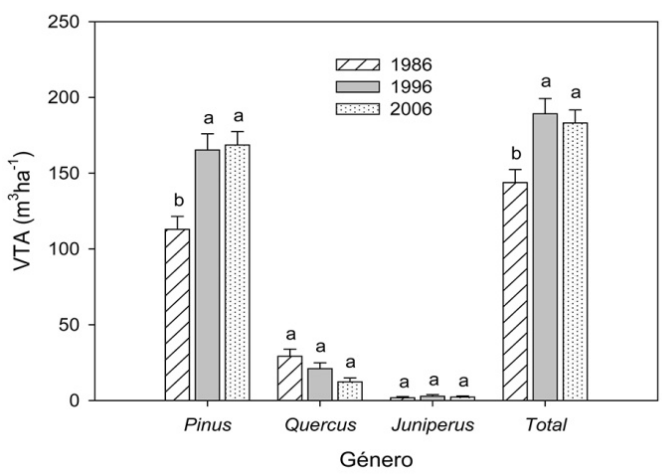

Figura 1. A) Número de árboles por ha por género y total. B. Área basal por género y total $\left(\mathrm{m}^{2} \mathrm{ha}^{-1}\right)$; C. Diámetro cuadrático por género y total $(\mathrm{cm})$; D. Altura promedio de Lorey, por género y total $\left(\mathrm{H}_{\mathrm{L}}\right)$. E. Volumen total árbol por género y total $\left(\mathrm{m}^{3} \cdot \mathrm{ha}^{-1}\right)$.

Valores promedios \pm error estándar. Letras diferentes $(a, b, c)$ indican diferencia significativa $(P<0.05)$. 
La prueba K-W no mostró diferencias significativas en el Dg de Quercus $\left(\mathrm{Chi}^{2}=0.80 ;\right.$ g.l. $\left.=2 ; \mathrm{P}=0.670\right)$, pero si en Juniperus $\left(\mathrm{Chi}^{2}\right.$ $=13.44 ;$ g.l. $=2 ; \mathrm{P}=0.001)$. En ambos géneros, los diámetros promedio de 1986, de $23.97 \mathrm{~cm} \pm 9.6 \mathrm{~cm}$ y el de 1996, de 17.81 $\mathrm{cm} \pm 5.19 \mathrm{~cm}$, respectivamente, se pueden considerar representativos (Fig. 1C).

\section{Altura media de Lorey $(\mathrm{HL}, \mathrm{m})$}

La $\mathrm{H}_{\mathrm{L}}$ del estrato arbóreo incrementó 19.7\% (2.06 m) entre 1986 y 2006; el $62.6 \%(1.29 \mathrm{~m})$ correspondió a la primera década, mostrando diferencias significativas $(\mathrm{F}=9.721 ;$ g.l. $=2 ; \mathrm{P}<$ 0.001) entre mediciones (Fig. 1D). $\mathrm{La} \mathrm{H}_{\mathrm{L}}$ media de 1986, $10.48 \mathrm{~m}$ $\pm 2.58 \mathrm{~m}$, fue diferente a las de $1996,11.77 \mathrm{~m} \pm 2.16 \mathrm{~m}$, y 2006, $12.54 \mathrm{~m} \pm 2.03 \mathrm{~m}$. El género Pinus incrementó $21.26 \%$ (2.22 m) entre 1986 y 2006; el $69.4 \%$ correspondió al primer intervalo (Fig. 1D). $\mathrm{La}_{\mathrm{L}}$ presentó diferencias significativas ( $\mathrm{F}=9.685$; g.l. $=2 ; \mathrm{P}<0.001)$ entre mediciones, la media de $1986,10.44 \mathrm{~m} \pm$ $2.91 \mathrm{~m}$, difirió de las medias de 1996, $11.98 \mathrm{~m} \pm 2.37 \mathrm{~m}$, y 2006, $12.66 \mathrm{~m} \pm 2.08 \mathrm{~m}$. En Quercus no reveló diferencias significativas $(\mathrm{F}=0.471 ;$ g.l. $=2 ; \mathrm{P}=0.626$ ), un valor representativo es la media de 2006, $10.60 \mathrm{~m} \pm 3.27 \mathrm{~m}$. En Juniperus, la prueba K-W rindió diferencias significativas $\left(\mathrm{Chi}^{2}=10.47\right.$; g.l. $\left.=2 ; \mathrm{P}=0.005\right)$.

\section{Altura dominante (Ho, m)}

La Ho promedio en el género Pinus incrementó $2.12 \mathrm{~m}$ en el periodo evaluado de 20 años y $76.9 \%$ tuvo lugar en el primer intervalo (Fig. 1E). La prueba $\mathrm{K}-\mathrm{W}$ indicó diferencias significativas $\left(\mathrm{Chi}^{2}=13.24 ;\right.$ g.l. $\left.=2 ; \mathrm{P}=0.001\right)$ entre las evaluaciones, y la prueba de comparaciones múltiples de Nemenyi determinó que la Ho media de 1986, $13.01 \mathrm{~m}$ (3.85 m), fue diferente respecto a 1996, $14.64 \mathrm{~m}$ (3.11 m), y 2006, $15.13 \mathrm{~m}$ (2.49 $\mathrm{m})$. La mediana de Ho incrementó $3 \mathrm{~m}$ en el periodo de 20 años. Respecto a la Ho de Quercus, el ANOVA no mostró diferencias significativas entre medias $(\mathrm{F}=0.460 ;$ g.l. $=2 ; \mathrm{P}=0.633)$. En el género Juniperus, la prueba K-W determinó la existencia de diferencias significativas en $\mathrm{Ho}\left(\mathrm{Chi}^{2}=8.85\right.$; g.l. $\left.=2 ; \mathrm{P}=0.012\right)$, entre los años 1986 y 2006. En la Ho total del bosque, el ANOVA no registró diferencias significativas $(\mathrm{F}=2.261 ;$ g.l. $=2 ; \mathrm{P}=$ 0.108); no obstante, numéricamente la media incrementó $1.37 \mathrm{~m}$ en el periodo de 20 años, lo que se debe probablemente a que en el cálculo se consideraron los árboles de mayor diámetro, sin importar el género.

\section{Volumen total árbol (VTA, m3·ha-1)}

El VTA fue evaluado antes de la corta. La masa arbórea alcanzó su máximo en 1996 y luego decreció, obteniendo un aumento final de 27.3\% (Fig. 1F). El ANOVA indicó diferencias significativas $(\mathrm{F}=7.258$; g.l. $=2 ; \mathrm{P}=0.001)$, entre el volumen de $1986,143.82 \mathrm{~m}^{3} \cdot \mathrm{ha}^{-1} \pm 58.34 \mathrm{~m}^{3} \cdot \mathrm{ha}^{-1}$, con respecto al de 1996 , $189.20 \mathrm{~m}^{3} \cdot \mathrm{ha}^{-1} \pm 68.60 \mathrm{~m}^{3} \cdot \mathrm{ha}^{-1}$, y $2006,183.12 \mathrm{~m}^{3} \cdot \mathrm{ha}^{-1} \pm 58.51$ $\mathrm{m}^{3} \cdot \mathrm{ha}^{-1}$. En Pinus, el VTA conservó una tendencia creciente, a diferencia de los otros géneros; aumentó $49.4 \%$ entre la primera y última medición, mostró diferencias significativas ( $F=11.020$; g.l. $=2 ; \mathrm{P}<0.001)$ entre monitoreos; en 1986 registró 112.85 $\mathrm{m}^{3} \cdot \mathrm{ha}^{-1} \pm 57.97 \mathrm{~m}^{3} \cdot \mathrm{ha}^{-1}$, que difirió del de 1996, $165.3 \mathrm{~m}^{3} \cdot \mathrm{ha}^{-1} \pm$ $72.60 \mathrm{~m}^{3} \cdot \mathrm{ha}^{-1}$, y $2006,168.60 \mathrm{~m}^{3} \cdot \mathrm{ha}^{-1} \pm 60.30 \mathrm{~m}^{3} \cdot \mathrm{ha}^{-1}$. El volumen de Quercus no presentó diferencias significativas $\left(\mathrm{Chi}^{2}=5.00\right.$; g.l. $=2 ; \mathrm{P}=0.082)$ entre monitoreos, aunque decreció $57.8 \%$. En Juniperus no existieron diferencias $\left(\mathrm{Chi}^{2}=0.14\right.$; g.l. $=2 ; \mathrm{P}=$ 0.934).

\section{Espaciamiento y espesura (a, $\mathrm{m} ; \mathrm{S}, \%)$}

Solo se evaluó para el arbolado total y Pinus, ya que Quercus y Juniperus no estaban presentes en varias parcelas. En esta variable no se observaron diferencias significativas en el bosque $\left(\mathrm{Chi}^{2}=\right.$ 1.53; g.l. $=2 ; \mathrm{P}=0.466)$, ni en Pinus $\left(\mathrm{Chi}^{2}=2.11\right.$; g.l. $=2 ; \mathrm{P}=$ $0.348)$ entre evaluaciones; sus promedios en el año 2006 fueron $30.1 \% \pm 8.29 \%$ para bosque y de $30.97 \% \pm 9.75 \%$ para Pinus y la menor espesura se registró en 1986, con 30.80\% $\pm 13.07 \%$ y $36.22 \% \pm 17.59 \%$, respectivamente. El índice de espaciamiento o espesura $(\mathrm{S} \%)$ no presentó diferencias significativas en Pinus $\left(\mathrm{Chi}^{2}=2.11 ;\right.$ g.l. $\left.=2 ; \mathrm{P}=0.348\right)$, ni en todo el arbolado $\left(\mathrm{Chi}^{2}=\right.$ 1.53; g.l. $=2 ; \mathrm{P}=0.466)$, entre evaluaciones. El nivel de significancia utilizado fue $\alpha=0.05$ (Tabla 1 ). 
Tabla 1. Espaciamiento promedio entre árboles en distribución a tresbolillo y espesura relativa de Pinus y la población total.

\begin{tabular}{|c|c|c|c|c|c|c|c|}
\hline \multirow{3}{*}{ ভ் } & \multirow[b]{3}{*}{ Par.\año } & \multicolumn{6}{|c|}{ Variables dasométricas } \\
\hline & & \multicolumn{3}{|c|}{$a(m)$} & \multicolumn{3}{|c|}{$\mathrm{S} \%$} \\
\hline & & 1986 & 1996 & 2006 & 1986 & 1996 & 2006 \\
\hline \multirow{8}{*}{$\stackrel{n}{\vdots}$} & Media & 4.31 & 4.23 & 4.57 & 36.22 & 30.16 & 30.97 \\
\hline & EE & 0.21 & 0.17 & 0.19 & 2.59 & 1.56 & 1.44 \\
\hline & $\mathrm{DE}$ & 1.46 & 1.17 & 1.27 & 17.59 & 10.60 & 9.75 \\
\hline & CV (\%) & 33.80 & 27.73 & 27.75 & 48.57 & 35.13 & 31.49 \\
\hline & Mediana & 4.13 & 4.04 & 4,34 & 32.13 & 28.81 & 30.14 \\
\hline & Mínimo & 2.21 & 2.02 & 2.22 & 15.15 & 12.56 & 16.47 \\
\hline & Máximo & 8.24 & 6.54 & 9.42 & 89.00 & 56.42 & 62.91 \\
\hline & $n$ & 46 & 46 & 46 & 46 & 46 & 46 \\
\hline \multirow{8}{*}{$\begin{array}{l}\bar{\pi} \\
\text { O } \\
\vdash\end{array}$} & Media & 3.84 & 3.90 & 4.40 & 30.80 & 28.01 & 30.10 \\
\hline & $\mathrm{EE}$ & 0.13 & 0.13 & 0.15 & 1.93 & 1.33 & 1.22 \\
\hline & $\mathrm{DE}$ & 0.91 & 0.85 & 1.02 & 13.07 & 9.03 & 8.29 \\
\hline & CV (\%) & 23.82 & 21.86 & 23.24 & 42.43 & 32.24 & 27.56 \\
\hline & Mediana & 3.90 & 3.92 & 4.31 & 28.07 & 26.63 & 29.84 \\
\hline & Mínimo & 2.16 & 2.01 & 2.20 & 12.08 & 12.06 & 17.02 \\
\hline & Máximo & 5.37 & 5.83 & 6.94 & 70.82 & 52.96 & 51.26 \\
\hline & $n$ & 46 & 46 & 46 & 46 & 46 & 46 \\
\hline
\end{tabular}

Nota: a, es el espaciamiento o distancia promedio entre árboles en distribución a tresbolillo; $\mathrm{S}_{\%}$, es la espesura relativa.

\section{DISCUSIÓN}

\section{Densidad}

En coincidencia con el estudio realizado por Serrada (2008), el N del bosque descendió con el paso del tiempo y el crecimiento de los árboles aumentó la proximidad entre ellos, por lo cual se aplicaron dos intervenciones silvícolas para evitar la mortalidad natural. Según Gadow et al. (2007), en los aclareos se eliminan individuos que compiten con los árboles deseables o de futura cosecha, por lo que el $\mathrm{N}$, como en este bosque, es uno de los parámetros más significativos en la conducción del crecimiento de un rodal (Gadow y Hui, 2001).

\section{Área basal}

El total del bosque y Pinus no recuperaron su nivel previo de $G$ en el año 2006, quizá por la corta de aclareo que provocó un descenso de $\mathrm{N}$ mayor al del periodo anterior que, si es intenso, afecta la recuperación de la masa (Prodan et al., 1997); aunado al descenso en la tasa de crecimiento diamétrico y probable menor reclutamiento. Según Prodan et al. (1997), el G tiene fuerte relación con la densidad y el volumen, y su incremento. Gadow et al. (2007) señalan que esto se puede observar en el comportamiento similar del G y VTA, y la relación directa entre el menor crecimiento en $\mathrm{G}$ con la menor densidad, $\mathrm{N}$ en el año 2006.

\section{Diámetro cuadrático}

Según Gadow y Hui (2001), los aclareos aplicados a bosques secundarios jóvenes, como el estudiado, favorecen el incremento en diámetro, de ahí que después de dos intervenciones silvícolas hubo incremento del Dg, en Pinus, Juniperus y en el total. En las tres evaluaciones, el valor del Dg promedio de Pinus y total fue similar a la mediana, según Prodan et al. (1997) y Bettinger et al. (2009), y esto ocurre en rodales con distribuciones diamétricas simétricas, típicas de masas regulares. 
Las diferencias significativas en Dg, de Pinus y total, avalan su incremento sostenido, lo que supone que los tratamientos silvícolas fueron favorables, ya que, según Gadow et al. (2007), la distribución diamétrica no solo está determinada por el crecimiento de los árboles sino también por las intervenciones silvícolas.

\section{Altura media de Lorey}

En masas regulares, la altura media incrementa hasta la edad madura, en tanto que la densidad $(\mathrm{N})$ declina (Bettinger, et al., 2009). Este comportamiento es evidente con las diferencias significativas del crecimiento en altura de Pinus y total y el descenso de la densidad en ambos, ratificando que la masa estudiada es regular y está en desarrollo. Asimismo, existe una relación directa entre la producción total en volumen de un rodal y su altura media, sin considerar la calidad de estación (DiéguezAranda et al., 2009), en la masa forestal estudiada se confirma esta relación $\mathrm{H}_{\mathrm{L}}-\mathrm{VT}$ T, específicamente para Pinus, en las diferentes evaluaciones.

\section{Altura dominante (Ho, m)}

A una misma edad, determinada especie desarrolla mayor altura en una estación de buena calidad que en una de mala, y, cuando aumenta la competencia entre los individuos de una masa, se da la diferenciación en tamaños de dominantes y dominados (Diéguez-Aranda et al., 2009). La altura dominante es una variable importante en las masas coetáneas; su crecimiento es prácticamente independiente de la densidad del rodal y no se afecta por las cortas, por lo que se usa para caracterizar el desarrollo de una masa y estimar el potencial productivo de un sitio (Gadow y Hui, 2001). En este estudio el crecimiento fue menor en el segundo intervalo, probablemente por la extracción de árboles dominantes o debido a su etapa de crecimiento.

\section{Volumen total árbol}

Según Návar y González (2009), la productividad en volumen aumenta con el índice de mezcla de Pinus y Quercus, y con el incremento en la densidad en bosques de Durango, México. En el presente estudio, se encontró que el aumento en proporción

(N) de Pinus (96.1\%) en 2006 generó un incremento del volumen corriente $(2 \%)$, inferior al del periodo anterior (46.4\%), cuando su proporción era menor (89.9\% en 1996) y existía mayor mezcla; aunque en este caso la densidad disminuyó y se desconoce el valor al inicio de cada periodo.

Los valores de G y VTA son previos a la corta; en las remediciones están afectados por el aprovechamiento y el reclutamiento, ambos no cuantificados, por lo que las diferencias entre evaluaciones no son necesariamente incrementos; en ocasiones decrecen, por lo que no es posible determinar el rendimiento neto de la masa. En la última evaluación, el género Pinus no recuperó su área basal, pero sí su volumen previo.

En el total del bosque y en Pinus, entre las dos últimas evaluaciones de $V T A$, no se encontró diferencia significativa; Pinus incrementó $2.0 \%$ y el total descendió $3.2 \%$, por la merma de Quercus (-41.5\%), lo que indica que el rendimiento no incrementó, sino relativamente se mantuvo, con una producción maderable sostenida. El VTA de Pinus en las dos remediciones superó los valores previos e incrementó su proporción con respecto al total del bosque: de 78.5\% en 1986 pasó a 92.1\% en 2006, y, en este último año, el VTA de Quercus representó 6.7\% y el de Juniperus $1.2 \%$ del total.

\section{Espaciamiento y espesura}

La ausencia de cambios significativos en $\mathrm{S} \%$, denota un adecuado manejo de la masa forestal; su valor más bajo (mayor espesura) se registró en 1996 (28.01\% y 30.16 \%), lo cual coincide con la mayor $\mathrm{G}$ en ambos, y con el VTA para el bosque total. DiéguezAranda et al. (2009) proponen mantener constante una espesura de 20\% en plantaciones de Pseudotsuga menziesii, para bosques naturales de Quercus robur de $23.3 \%$ y para Betula alba de 30\%, para propiciar el crecimiento en diámetro en una región de España. Estos valores son antes del aclareo, después de la intervención; para B. alba, proponen dejar un S\% de 40\%. Chinchilla et al. (2011) probaron índices de espesura de 21\%,23\% y 25\% en plantaciones de Cupressus lusitanica en Costa Rica; los mejores incrementos para ciertos parámetros, localidad y edad, los obtuvieron con $25 \%$. Según Serrada (2008), en los bosques naturales de Norteamérica el silvicultor debe reducir la espesura para aumentar el crecimiento individual y mejorar la productividad. 


\section{CONCLUSIONES}

El bosque en conjunto y el género Pinus en particular tuvieron una dinámica de crecimiento y estabilidad en $G$, incrementaron en forma sostenida en $\mathrm{Dg}$ y $\mathrm{H}_{\mathrm{L}}$; en volumen difirieron en la última evaluación. La N del bosque, Pinus y Quercus disminuyó, pero la variable espesura se mantuvo. El género Quercus decreció en sus parámetros, excepto en $\mathrm{Dg}$, por efecto del manejo silvícola orientado a la productividad maderable, que favoreció a Pinus por su valor económico; Juniperus incrementó en dimensiones, pero en los valores de densidad fue constante. A partir del comportamiento de los parámetros dasométricos del bosque, se infiere que el rendimiento maderable incrementó en el primer periodo y en el segundo se sostuvo. Entre parcelas y rodales se mantuvo la heterogeneidad, pero el bosque conservó homogeneidad; esta dinámica de crecimiento general de los parámetros dasométricos corresponde a un bosque regular, que no puede mantenerse de manera permanente, por lo que, se cree, deberá replantearse la gestión forestal a largo plazo, con el propósito de mantener la producción y lograr una gestión forestal sustentable. En general, el bosque analizado incrementó su volumen y dimensiones del arbolado, y mantuvo su producción maderable con el aprovechamiento de las cortas intermedias, necesarias para su crecimiento.

\section{REFERENCIAS}

Aguirre, O. A., Hui, G., Gadow, K., \& Jiménez, J. (2003). An analysis of spatial forest structure using neighbourhood-based variables. Forest Ecology and Management, 183(1-3), 137-145. doi: 10.1016/S0378-1127(03)00102-6.

Bengoa, J. L. (1999). Estimación de la altura dominante de la masa a partir de la "altura dominante de parcela". Ventajas frente a la altura dominante de Assman. Investigación Agraria: Sistemas y Recursos Forestales, 8(3), 311-321.

Bell, F. W., Hunt, S., Dacosta, J., Sharma, M., Larocque, G. R., Winters, J. A., \& Newmaster, S. G. (2014). Effects of silviculture intensity on plant diversity response patterns in young managed northern temperate and boreal forests. Écoscience, 21(3-4), 327-339. doi: 10.2980/21-(3-4)-3710.

Bettinger, P., Boston, K., Siry, J. P., \& Grebner, D. L. (2009). Forest management and planning. San Diego, Estados Unidos: Academic Press.

Chinchilla, O., Chaves, E., \& Mora, F. (2011). Comparación de crecimientos bajo diferentes intensidades de manejo en plantaciones de ciprés (Cupressus lusitanica Miller) en dos sitios de Costa Rica. Revista Baracoa, 30, 1-19.
Comisión Nacional para el Conocimiento y Uso de la Biodiversidad [Conabio] (Cartógrafo). (1998). Clasificación climática. Recuperado de http://infoteca.semarnat.gob.mx/

Corral, J. J., Aguirre, O. A., Jiménez, J., \& Corral, S. (2005). Un análisis del efecto del aprovechamiento forestal sobre la diversidad estructural en el bosque mesófilo de montaña «El Cielo», Tamaulipas, México. Investigación Agraria: Sistemas y Recursos Forestales, 14(2), 217-228.

Diéguez-Aranda, U., Rojo-Alboreca, A., Castedo-Dorado, F., Álvarez González, J. G., Barrio-Anta, M., Crecente-Campo, F., \& Sánchez Rodríguez, F. (2009). Herramientas selvicolas para la gestión forestal sostenible en Galicia. Lugo, España: Tórculo Artes Gráficas, S.A.

Fry, B. P. (2011). Community forest monitoring in REDD+: the ' $\mathrm{M}$ ' in MRV? Environmental Science and Policy, 14(2), 181-187. doi: 10.1016/j.envsci.2010.12.004

Gadow, K., \& Hui, G. (2001). Modelling stand development. En Gadow, K. H. (ed.), Modelling Forest Development (pp. 26-60). Dordrecht, Países Bajos: Kluwer academic pub.

Gadow, K., Sánchez, O. S., \& Álvarez, G. J. G. (2007). Estructura y crecimiento del bosque. Göttingen, Alemania: Universidad de Göttingen.

García-Gonzalo, J., Zubizarreta-Gerendiain, A., Kellomäki, S., \& Peltola, H. (2017). Effects of forest age structure, management and gradual climate change on carbon sequestration and timber production in finnish boreal forests. En Managing Forest Ecosystems: The Challenge of Climate Change (pp. 277-298). Springer International Publishing. doi: 10.1007/978-3-319-28250-3_14.

Graciano-Ávila, G., Aguirre-Calderón, O. A., Alanís-Rodríguez, E., \& Lujan-Soto, J. E. (2017). Composición, estructura y diversidad de especies arbóreas en un bosque templado del noroeste de México. Ecosistemas y Recursos Agropecuarios, 4(12), 535-542. doi: 10.19136/era.a4n12.1114.

Hernández-Salas, J., Aguirre-Calderón1, O. A., Alanís-Rodríguez, E., Jiménez-Pérez1, J., Treviño-Garza1, E. J., González-Tagle1, M. A., Luján-Álvarez, C., Olivas-García, J. M., \& Domínguez-Pereda, L. A. (2013). Efecto del manejo forestal en la diversidad y composición arbórea de un bosque templado del noroeste de México. Chapingo serie Ciencias Forestales y del Ambiente, 19(3), 189199. doi: 10.5154/r.rchscfa.2012.08.052.

Instituto Nacional de Estadística y Geografía [Inegi] (Cartógrafo). (1984). Carta de uso de suelo y vegetación, H12-9 (Madera). México: Inegi.

Manzanero, M., \& Pinelo, G. (2004). Plan silvicultural en unidades de manejo forestal Reserva de la Biosfera Maya, Petén, Guatemala. San Francisco de Dos Ríos, Costa Rica: Fondo Mundial para la Naturaleza y PROARCA.

Jiménez, J., Alanís, E., González, M. A., Aguirre, O. A., \& Treviño, E. J. (2013). Characterizing regeneration of woody species in areas with different land-history tenure in the Tamaulipan thornscrub, Mexico. The Southwestern Naturalist, 58(3), 299-304. doi: 10.1894/0038-4909-58.3.299.

López, J. A., Aguirre-Calderón, O. A., Alanís-Rodríguez, E., Monarrez, J. C., González-Tagle, M. A., \& Jiménez-Pérez, J. (2017). Composición y diversidad de especies forestales en bosques templados de puebla, México. Madera y Bosques, 23(1):39-51. doi: $10.21829 /$ myb.2017.2311518. 
McGinley, K., \& Finegan, B. (2001). Criterios e indicadores para evaluar la sostenibilidad ecológica: un conjunto integrado para bosques manejados en Costa Rica. Revista Forestal Centroamericana, (34), 2327.

Návar, J. J., \& González, G. S. (2009). Diversidad, estructura y productividad de bosques templados de Durango, México. Polibotánica, (27), 71-87.

Prodan, M., Peters, R., Cox, F., \& Real, P. (1997). Mensura forestal. San José, Costa Rica: IICA-BMZ/GTZ. Serie Investigación y Educación en Desarrollo Sostenible.

Reque, J., Sarasola, M., Gyenge, M., \& Fernández, M. E. (2007). Caracterización silvícola de ñirantales del norte de la Patagonia para la gestión forestal sostenible. Bosque, 28(1), 33-45.

Serrada, H. R. (2008). Apuntes de selvicultura. En Universidad Pontificia de México (ed.), S.-p. Didácticas (Series Ed.). México: Servicio de Publicaciones, UPM.

SmartWood. (2004). Resumen público de certificación de ejido El Largo y anexos. En CCMSS (ed.), (pp. 40). Nueva York, Estados Unidos: SmartWood.
Wheater, C. P., \& Cook, P. A. (2005). Using statistics to understand the environment (Routledge, Taylor \& Francis e-Library ed.). Londres, Inglaterra: Routledge.

Manuscrito recibido el: 14 de diciembre de 2015

Aceptado el: 18 de octubre de 2017

Publicado el: 18 de junio de 2018

Este documento se debe citar como:

Hernández-Salas, J., Aguirre-Calderón, O. A., Alanís-Rodríguez, E., Jiménez-Pérez, J., Treviño-Garza, E. J., González-Tagle, M. A., LujánÁlvarez, C., Olivas-García, J. M., \& Domínguez-Pereda, L. A. (2O18). Dinámica del crecimiento de un bosque templado bajo manejo en el noroeste de México. Madera y Bosques, 24(2), e2421767. doi: $10.21829 /$ myb.2018.2421767

Madera y Bosques por Instituto de Ecología, A.C. se distribuye bajo una Licencia Creative Commons Atribución-NoComercialCompartirlgual 4.0 Internacional. 